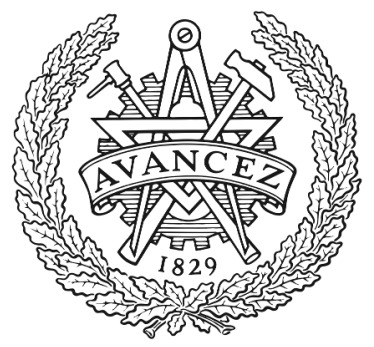

CHALMERS

UNIVERSITY OF TECHNOLOGY

\title{
A combined theoretical and experimental study of the polymer matrix mediated stress transfer in a cellulose nanocomposite
}

Downloaded from: https://research.chalmers.se, 2023-04-26 11:39 UTC

Citation for the original published paper (version of record):

Peterson, A., Mehandzhiyski, A., Svenningsson, L. et al (2021). A combined theoretical and experimental study of the polymer matrix mediated stress transfer in a cellulose nanocomposite. Macromolecules, 54(7): 3507-3516.

http://dx.doi.org/10.1021/acs.macromol.0c02305

N.B. When citing this work, cite the original published paper. 


\section{A Combined Theoretical and Experimental Study of the Polymer Matrix-Mediated Stress Transfer in a Cellulose Nanocomposite}

Anna Peterson, Aleksandar Y. Mehandzhiyski, Leo Svenningsson, Agnieszka Ziolkowska, Roland Kádár, Anja Lund, Linda Sandblad, Lars Evenäs, Giada Lo Re,* Igor Zozoulenko,* and Christian Müller*

Cite This: Macromolecules 2021, 54, 3507-3516

Read Online

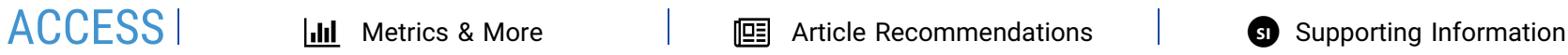

ABSTRACT: We study composites of cellulose nanocrystals (CNCs) in an ionomer matrix of poly(ethylene-stat-sodium acrylate) and find that direct cellulose/cellulose interactions in the composite are not a requirement for achieving reinforcement. While isotropic composites only show a slightly enhanced stiffness compared to the neat ionomer, a more substantial increase in Young's modulus by a factor of up to 5 is achieved by uniaxial alignment of the composites through melt spinning. The orientation of $\mathrm{CNC}$ in melt-spun composites reduces the probability of cellulose/

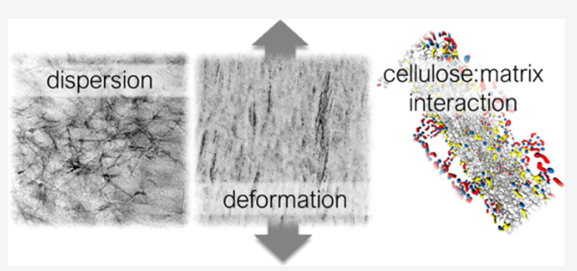
cellulose interactions, which suggests that cellulose/polymer interactions must be present that lead to the observed reinforcement. Molecular dynamics simulations confirm strong cellulose/polymer interactions in the form of ionic interactions as well as hydrogen bonding. These cellulose/polymer interactions facilitate efficient stress transfer, leading to the high reinforcing effect of $\mathrm{CNC}$, while cellulose/cellulose interactions play a minor role in the mechanical response of the composite.

\section{INTRODUCTION}

In the strive for mechanically strong and stiff materials, which are also lightweight and originate from renewable sources, cellulose fibers and their nanoscale derivatives receive widespread attention. ${ }^{1-5}$ Hydrolysis of refined cellulosic fibers liberates cellulose nanocrystals (CNCs), highly crystalline, elongated particles with a diameter of a few nanometers. ${ }^{6}$ The high stiffness in combination with its low density gives $\mathrm{CNC}$ a high specific modulus of about $90 \mathrm{MPa} \mathrm{kg}{ }^{-1} \mathrm{~m}^{-3}$, which is comparable to the specific modulus of clay platelets. ${ }^{8}$ The critical challenge that must be met to utilize the full potential of CNC and other nanocelluloses in composites is to transfer the exceptional properties from a single cellulose crystal to the bulk nanocomposites. Knowledge about the mechanism of stress transfer is integral for the optimized utilization of nanocelluloses in composite materials. The two main modes of stress transfer that can occur in a CNC nanocomposite are (1) load sharing between the matrix and nanocellulose ${ }^{1}$ and (2) the nanocellulose forms a load-bearing network governed by cellulose/cellulose contacts and transmission of force via the matrix plays a reduced role. ${ }^{9,10}$ The formation of a continuous network of a stiff reinforcing agent, such as CNC, will drastically increase the elastic modulus of a low modulus, rubbery polymer, which is well described in the cellulose literature. ${ }^{10-16}$

A number of publications have highlighted the dilemma present in the manufacturing of cellulose nanocomposites: ${ }^{9,17-20}$ a homogenous dispersion and minimal aggregation of the nanocellulose demand favorable cellulose/polymer interactions, while formation of a percolated network of cellulose requires favorable cellulose/cellulose interactions. Aitomäki and Oksman ${ }^{21}$ reviewed the reinforcing efficiency of nanocelluloses in numerous matrices and concluded that very few composites showed a mechanical response indicative of force transfer through the cellulose/cellulose contact. It has been argued $^{9}$ that efforts to improve dispersion and decrease self-aggregation of nanocelluloses in nonpolar matrices by various surface treatments, such as surfactant adsorption, ${ }^{22,23}$ hydrophobization, ${ }^{23,24}$ or grafting of polymer chains, ${ }^{25}$ also shield cellulose/cellulose interactions. Hence, cellulose cannot percolate, and the desired network is not formed. A high degree of surface modification decreases the strength of interaction between cellulose particles, ${ }^{26}$ and therefore, any enhancement in stiffness, which has been observed in a number of composites, $9,17-20,22,24,27$ possibly occurred due to improved interactions between cellulose and the polymer matrix rather than the formation of a percolated cellulose network.

Here, we employ solvent casting of native CNC with a water-borne polymer latex of the ionomer poly(ethylene-statsodium acrylate) $\left(\mathrm{EAA}_{15}\right)$. The excellent dispersion attained using this water-based mixing technique ${ }^{10,11,28,29}$ makes such composites interesting systems to elucidate the mechanism of

Received: October 8, 2020

Revised: February 5, 2021

Published: March 19, 2021 
stress transfer, i.e., to discern if forces are transmitted via a continuous cellulose network or if cellulose/polymer interactions are responsible for the reinforcing effect. Further, the $\mathrm{EAA}_{15}$ polymer has a comparably high stiffness $(235 \mathrm{MPa})$, relative to matrices in which cellulose networks have previously shown a strong reinforcing effect (typical polymer stiffness $<10$ $\mathrm{MPa})$. Hence, the stress transfer behavior in the $\mathrm{CNC} / \mathrm{EAA}_{15}$ composite has interesting implications to judge the potential of reinforcement by a CNC network in other semicrystalline matrices. We carry out a detailed experimental investigation of a series of isotropic samples produced by water-assisted mixing, casting from the obtained suspension and subsequent compression molding. Further, we study anisotropic samples produced by melt spinning, where orientation of $\mathrm{CNC}$ reduces the number of possible cellulose/cellulose contacts. We conclude that the cellulose network present in the isotropic samples does not have a decisive impact on the composite modulus, but stress transfer via cellulose/polymer interactions has to be considered to explain the reinforcement in this type of cellulose nanocomposite. We also perform molecular dynamics (MD) simulations to gain detailed insight into the makeup of the cellulose/polymer interface and find a lowdensity polymer phase close to the CNC surface.

\section{EXPERIMENTAL SECTION}

Materials. $\mathrm{NaOH}$-neutralized cellulose nanocrystals (CNCs) from sulfuric acid hydrolysis were obtained as a spray-dried powder from CelluForce, Canada. CNC was dispersed in water at a solid content of $4 \%$ using an Ultra Turrax high shear mixer at $14000 \mathrm{rpm}$ for $8 \mathrm{~min}$. Poly(ethylene-stat-sodium acrylate), the $\mathrm{NaOH}$-neutralized form of poly(ethylene-stat-acrylic acid) comprising $15 \mathrm{wt} \%$ acrylic acid $\left(\mathrm{EAA}_{15}\right)$, had a density of $0.994 \mathrm{~g} \mathrm{~cm}^{-3}$ and a melt flow rate of $36 \mathrm{~g} /$ $10 \mathrm{~min}$ (ISO $1133,190^{\circ} / 2.16 \mathrm{~kg}$ ) and was obtained as an aqueous latex dispersion from BIMKemi AB, Sweden (20 wt \% solid content; $\mathrm{pH}$ adjusted to 9.7 with $\mathrm{NaOH}$ ).

Sample Preparation. CNC/EAA 15 composites were prepared by (1) mixing a freshly dispersed suspension of $\mathrm{CNC}$ with the $\mathrm{EAA}_{15}$ dispersion using an excess of water (total water content $96 \pm 1 \%$ ), (2) vigorous stirring for $10 \mathrm{~min}$, and (3) casting into polypropylene molds with subsequent drying at room temperature, which resulted in about $0.5 \mathrm{~mm}$ thick sheets with the desired CNC content. The dry composite sheets were milled in a rotor mill to obtain irregularly formed composite flakes, with sizes in the millimeter range. These flakes were pressed at $140{ }^{\circ} \mathrm{C}$ into plaques with a thickness of $500 \mu \mathrm{m}$. Unstretched extrudates were prepared by compounding the dried and milled composite material at $140^{\circ} \mathrm{C}$ using a twin-screw Xplore Micro Compounder, followed by extrusion at a constant force $(2-2.5 \mathrm{kN}$ depending on sample viscosity) and take-up of the extrudates onto a treadmill, while fibers were prepared by collection of the extrudate with an Xplore Micro Fiber Line using three different collector speeds $\left(0.1,5\right.$, and $\left.10 \mathrm{~m} \mathrm{~min}^{-1}\right)$ and a constant collector torque of $75 \mathrm{~N}$. CNC films were prepared by casting a freshly dispersed suspension of CNC (4 wt \% solid content) into polypropylene molds with subsequent drying at room temperature.

Extensional Rheology. Extensional rheology was performed using an Anton Paar MCR 702 TwinDrive rheometer equipped with an Anton Paar Universal Extensional Fixture at a temperature of 140 ${ }^{\circ} \mathrm{C}$ and Hencky strains of $0.1-20 \mathrm{~s}^{-1}$. It should be noted that the ductility of samples prohibited sample fracture inside the rheometer; hence, the full range of strain hardening was not attained.

Differential Scanning Calorimetry (DSC). DSC was carried out under nitrogen between -50 and $150{ }^{\circ} \mathrm{C}$ at a scan rate of $10{ }^{\circ} \mathrm{C}$ $\min ^{-1}$ using a Mettler Toledo DSC2 calorimeter equipped with a HSS7 sensor and a TC-125MT intercooler. The sample weight was 3-4 mg. Fractional crystallization was carried out according to a previously published procedure, ${ }^{30}$ i.e., annealing for $4 \mathrm{~h}$ at stepwise decreasing temperatures between 110 and $40{ }^{\circ} \mathrm{C}$.
Transmission Electron Microscopy (TEM). Specimens for TEM were sectioned under cryo conditions using a Leica EM FC7 cryo ultramicrotome with a Diatome cryoknife at $-120{ }^{\circ} \mathrm{C}$. Sections $(100$ $\mathrm{nm}$ thin) were placed on formvar-coated copper grids, stained with uranyl acetate, and air dried at room temperature. Imaging was performed using an FEI Talos L120 TEM and recorded with a FEI Ceta CMOS $4 \mathrm{k} \times 4 \mathrm{k}$ pixel detector.

Tensile Testing. Tensile testing was done with an Instron 5565A with a gauge length of $20 \mathrm{~mm}$ at a cross-head speed of $6 \mathrm{~mm} \mathrm{~min}^{-1}$.

Dynamic Mechanical Analysis (DMA). DMA thermograms were recorded between -70 and $30{ }^{\circ} \mathrm{C}$ at a heating rate of $3{ }^{\circ} \mathrm{C}$ $\min ^{-1}$, a frequency of $20 \mathrm{~Hz}$, and $0.1 \%$ strain using a Rheometrics Solids Analyzer RSA II. Prior to temperature sweeps, strain sweeps were carried out to ensure that the applied strain was within the linear viscoelastic region.

Thermogravimetric Analysis (TGA). TGA was carried out under nitrogen at a scan rate of $10{ }^{\circ} \mathrm{C} \mathrm{min}{ }^{-1}$ using a Mettler Toledo TGA/DSC 3+.

Molecular Dynamics Simulations. All-atom molecular dynamics (MD) simulations were carried out with the OPLS-AA force field $^{31,32}$ and the GROMACS v.2018 simulation package (see the Supporting Information for details). ${ }^{33}$

Wide-Angle X-ray Scattering (WAXS). WAXS diffractograms were obtained using a Mat:Nordic instrument from SAXSLAB equipped with a Rigaku 003+ high brilliance microfocus $\mathrm{Cu}$ radiation source (wavelength $=1.5406 \AA$ ) and a Pilatus $300 \mathrm{~K}$ detector placed about $43 \mathrm{~mm}$ from the sample.

Rotor-Synchronized Magic Angle Spinning (ROSMAS) Solid-State Nuclear Magnetic Resonance (NMR) Spectroscopy. NMR measurements were carried out using a Bruker $500 \mathrm{MHz}$ Avance III, operating at $125.8 \mathrm{MHz}$ for ${ }^{13} \mathrm{C}$. The ROSMAS experiment was conducted at ambient temperature and $1500 \pm 1$ $\mathrm{Hz}$ rotor rotation rate to sufficiently separate a few cellulose sidebands from other signals (see the Supporting Information for details).

\section{RESULTS AND DISCUSSION}

Preparation and Characterization of Isotropic CNC/ EAA $_{15}$ Composites. As the matrix material, we selected the ionomer $\mathrm{EAA}_{15}$ (Figure 1a), which has a polar comonomer

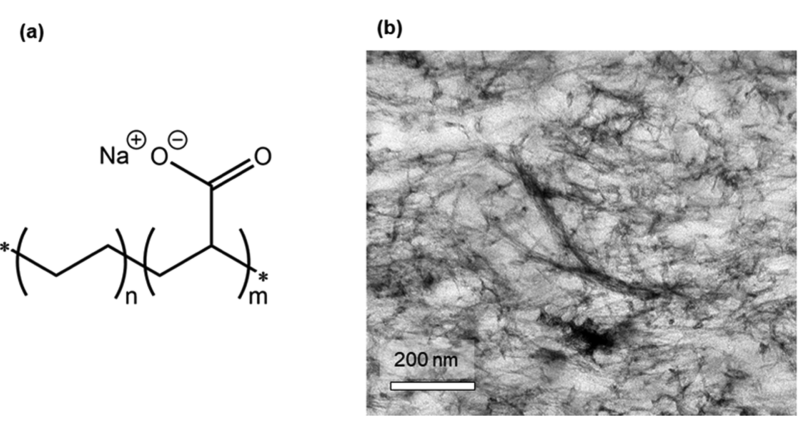

Figure 1. (a) Molecular structure of poly(ethylene-stat-sodium acrylate). (b) TEM image of a cryomicrotome-sectioned sample of a melt-pressed film of $\mathrm{CNC} / \mathrm{EAA}_{15}$ comprising $10.5 \mathrm{vol} \% \mathrm{CNC}$.

content of 15 wt \%. $\mathrm{EAA}_{15}$ is a branched polymer, as evidenced by extensional rheology (Figure S1), and features a statistical distribution of the polar comonomers, which are, on average, separated by $30-\mathrm{CH}_{2}-$ units (see Figure $\mathrm{S} 2$ for fractional crystallization of $\mathrm{EAA}_{15}$ ). We prepared composites of $\mathrm{EAA}_{15}$ with native $\mathrm{CNC}$ using water-assisted mixing, followed by casting from the obtained dilute water dispersion and finally compression molding of the dried and milled material into plaques. We note that the material did not experience any substantial shear during pressing, and therefore, we rule out 

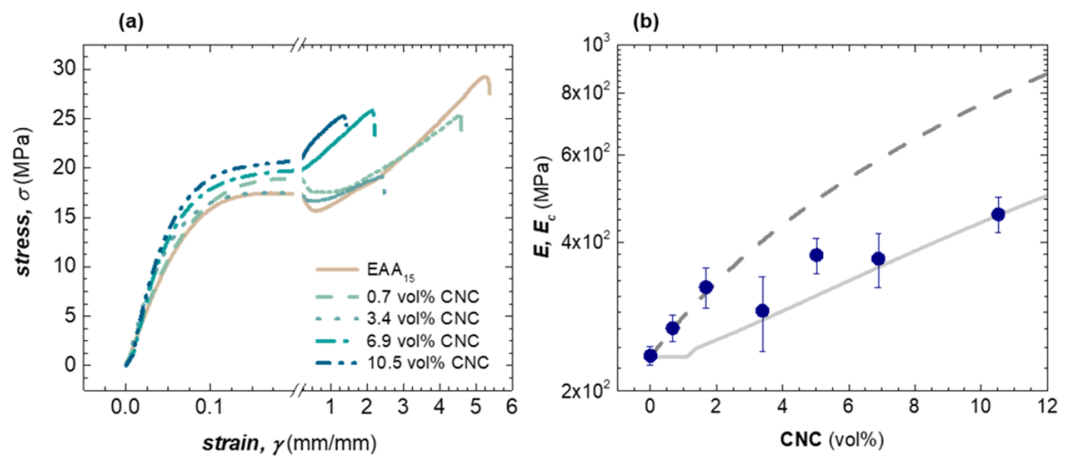

Figure 2. (a) Representative tensile stress-strain curves measured for neat $\mathrm{EAA}_{15}$ as well as composites with $0.7-10.5$ vol \% and (b) Young's modulus $E$ of isotropic composites (blue circles) as well as predicted moduli $E_{\mathcal{O}}$ predicted by the Halpin-Tsai model (dashed line) and Ouali model (solid line).

that this final preparation step influenced the distribution of the $\mathrm{CNC}$ reinforcing agent.

Transmission electron microscopy (TEM) images of cryo ultramicrotome cut thin sections of the obtained $\mathrm{CNC} / \mathrm{EAA}_{15}$ composite containing 10.5 vol \% CNC show well-dispersed CNC (Figure $1 \mathrm{~b}$ ), comprising mainly individually dispersed CNC (length $L=(300 \pm 100) \mathrm{nm}$; diameter $d=(5 \pm 2) \mathrm{nm}$; cf. Figure S3) together with a minor fraction of aggregates. A comparison of different areas indicates that the degree of dispersion is comparable for different sections of the sample (Figure S4). The recorded TEM images reveal a good dispersion of $\mathrm{CNC}$, which would allow the formation of a cellulose network. The percolation threshold, $V_{\mathcal{c}}$ of rodlike nanoparticles is linked to the aspect ratio via ${ }^{1}$

$$
V_{\mathrm{c}}=\frac{0.7}{L / d}
$$

where $L$ and $d$ are the length and diameter of the nanoparticle, respectively. Eq 1 suggests that the here-used $\mathrm{CNC}$, which has an aspect ratio of $L / d=60$, can percolate above a volume fraction of $1.2 \mathrm{vol} \%$.

Tensile Deformation of Isotropic Samples. We carried out tensile deformation experiments on specimens from isotropic, compression-molded composite films, having a CNC content of $0.7-10.5 \mathrm{vol} \%$ (Figure 2a; cf. the Supporting Information for calculation of vol \%). We compared the change in Young's modulus $E$ as a function of CNC content with values predicted by models that are commonly used to describe the behavior of cellulose nanocomposites (Figure $2 b$ ), keeping in mind the limitations of said models to correctly describe viscoelastic materials, as the theory behind them assumes completely elastic behavior of both matrix and reinforcement. We considered two models, the Halpin-Tsai and the Ouali model, which account for the geometry and elastic properties of the nanoscale reinforcing agent but differ in the way that interparticle interactions are treated. The former does not account for the stiffening effect from stress transfer via interparticle interactions, whereas the latter, which is a percolation model, considers interparticle interactions and their impact on the composite modulus. ${ }^{1}$ According to the Halpin-Tsai model, $E$ is given by ${ }^{34}$

$$
E=E_{\mathrm{m}} \frac{\left(1+\xi \eta V_{\mathrm{r}}\right)}{\left(1-\eta V_{\mathrm{r}}\right)}
$$

where

$$
\eta=\frac{\frac{E_{\mathrm{r}}}{E_{\mathrm{m}}}-1}{\frac{E_{\mathrm{r}}}{E_{\mathrm{m}}}+\xi}
$$

where $E_{\mathrm{m}}$ and $E_{\mathrm{r}}$ refer to Young's modulus of the matrix and the reinforcing agent, respectively, and $V_{\mathrm{r}}$ and $\xi / 2$ are the volume fraction and aspect ratio of the reinforcing agent. For the modulus of individual CNC particles, we used a literature value of $E_{\mathrm{r}}=130 \mathrm{GPa}$, representing the average reported modulus for a single cellulose I crystal. ${ }^{35}$ As such, the modulus predictions of the Halpin-Tsai model mark the upper bound for possible reinforcement, assuming perfect interfacial adhesion and dispersion, and using $E_{\mathrm{r}}$ of completely crystalline cellulose. Young's modulus of the matrix $E_{\mathrm{m}}=235 \mathrm{MPa}$ was measured by tensile deformation. Eq 2 can be extended to account for short fiber composites with randomly oriented fibers

$$
E=E_{\mathrm{m}}\left[A \frac{\left(1+\xi_{\|} \eta_{\|} V_{\mathrm{r}}\right)}{\left(1-\eta_{\|} V_{\mathrm{r}}\right)}+B \frac{\left(1+\xi_{\perp} \eta_{\perp} V_{\mathrm{r}}\right)}{\left(1-\eta_{\perp} V_{\mathrm{r}}\right)}\right]
$$

where $\eta_{\|}$and $\eta_{\perp}$ are calculated according to eq 3 with $\xi_{\|}=2 L /$ $d$ and $\xi_{\perp}=2$. $A$ and $B$ are coefficients related to the type of anisotropy. For composites that are isotropic in three dimensions, van $\mathrm{Es}^{36}$ has proposed $A=0.184$ and $B=0.816$.

For the Ouali model, the composite elastic modulus is given by $^{37,38}$

$$
E=\frac{\left(1-2 \psi+\psi V_{\mathrm{r}}\right) E_{\mathrm{m}} E_{\mathrm{n}}+\left(1-V_{\mathrm{r}}\right) \psi E_{\mathrm{n}}^{2}}{\left(1-V_{\mathrm{r}}\right) E_{\mathrm{n}}+\left(V_{\mathrm{r}}-\psi\right) E_{\mathrm{n}}}
$$

In the adaptation of the Ouali model commonly used for cellulose nanocomposites, ${ }^{11,14} E_{\mathrm{n}}$ is the elastic modulus of the reinforcing network, measured by tensile deformation of a neat CNC film. $E_{\mathrm{n}}=5.2 \mathrm{GPa}$ was measured by tensile deformation (see Experimental Section for details). $\psi$ denotes the volume fraction of the reinforcing agent that participates in the percolating network and is obtained from

$$
\psi= \begin{cases}0 & V_{\mathrm{f}} \leq V_{\mathrm{c}} \\ V_{\mathrm{r}}\left(\frac{V_{\mathrm{r}}-V_{\mathrm{c}}}{1-V_{\mathrm{c}}}\right)^{\mathrm{b}} & V_{\mathrm{f}}<V_{\mathrm{c}}\end{cases}
$$

where $b$ is the critical percolation exponent, with a value of 0.4 in the case of a three-dimensional (3D) network, $, 37,38$ and $V_{c}$ is taken from eq 1 . 
The trend of $E$ with CNC content predicted by the HalpinTsai model for 3D isotropic composites (eq 4) is in good agreement with our experimental data up to a CNC content of 1.7 vol \% (Figure $2 \mathrm{~b}$ ). At a higher CNC content, the predicted modulus is higher compared to the experimental values, which we explain with the onset of aggregation of $\mathrm{CNC}$, bearing in mind that the Halpin-Tsai model assumes perfect dispersion of the reinforcing agent. In contrast, the Ouali model (eq 6) underestimates $E$ at $\mathrm{CNC}$ concentrations of less than 1.7 vol $\%$, while it shows a relatively good agreement with the experimental data at a higher CNC content. It is worth noting that, as the matrix is comparably stiff in comparison to the reinforcing network, the Ouali model predicts that the onset of percolation does not lead to a drastic increase in the modulus. Considering the relatively small difference between predictions of composite stiffness using the two models, we conclude that fitting models to mechanical data alone is not sufficient to judge the major path of stress transfer in our composites.

Melt Spinning of Anisotropic Composite Fibers. To gain additional insight into the relative importance of cellulose/cellulose and cellulose/polymer interactions in our $\mathrm{CNC} / \mathrm{EAA}_{15}$ composites, we prepared anisotropic, oriented fibers by melt spinning (Figure S5). Our aim was to create a set of samples with a high degree of uniaxial alignment of the $\mathrm{CNC}$ reinforcing agent, which reduces the probability of cellulose/cellulose interactions. This type of behavior has been observed for, e.g., oriented carbon nanotube composites, which display a drastic reduction in electrical conductivity due to loss of percolation. ${ }^{39,40}$ Carbon fiber composites show similar behavior, with an increase in percolation threshold due to orientation. ${ }^{41}$ Samples with a draw ratio of 1 were produced by extrusion and subsequent take-up of the extrudate onto a treadmill, without stretching, while fibers with draw ratios of 2-40 were prepared by extrusion and subsequent solid-state drawing.

CNC Orientation in Anisotropic Samples. To visualize the alignment of $\mathrm{CNC}$, we prepared thin sections of anisotropic samples cut along the axis of orientation using a cryo ultramicrotome. TEM images of the $\mathrm{CNC} / \mathrm{EAA}_{15}$ composite with 10.5 vol \% CNC reveal striking differences between isotropic material and anisotropic samples, in the form of unstretched extrudate and melt-spun fibers (Figure 3). The orientation of CNC occurs already upon extrusion and is further enhanced by the draw down experienced during melt spinning. Wide-angle X-ray scattering (WAXS; Figure S7) and solid-state nuclear magnetic resonance (solid-state NMR) spectroscopy in combination with the rotor-synchronized magic angle spinning (ROSMAS) technique ${ }^{42,43}$ confirm that CNC orients already upon extrusion and ROSMAS NMR further shows that alignment of $\mathrm{EAA}_{15}$ requires solid-state drawing (Figures S8-S9).

The CNCs in the anisotropic samples display not only orientation but also an increased degree of aggregation with fewer individually dispersed CNC particles compared to the isotropic sample. It is worth noting that compounding, despite excellent dispersion preceding the melt processing step, can have adverse effects on CNC dispersion. ${ }^{9,44}$ Melt compounding has been reported to cause a reduction in the aspect ratio of nanocelluloses, ${ }^{45,46}$ to cause aggregation ${ }^{47}$ and to have a severe impact on the microstructure resulting in a significantly reduced tensile modulus. ${ }^{46}$ The here presented TEM images confirm that melt compounding of the composite impacts the dispersion of native CNC (Figure 3). We argue that
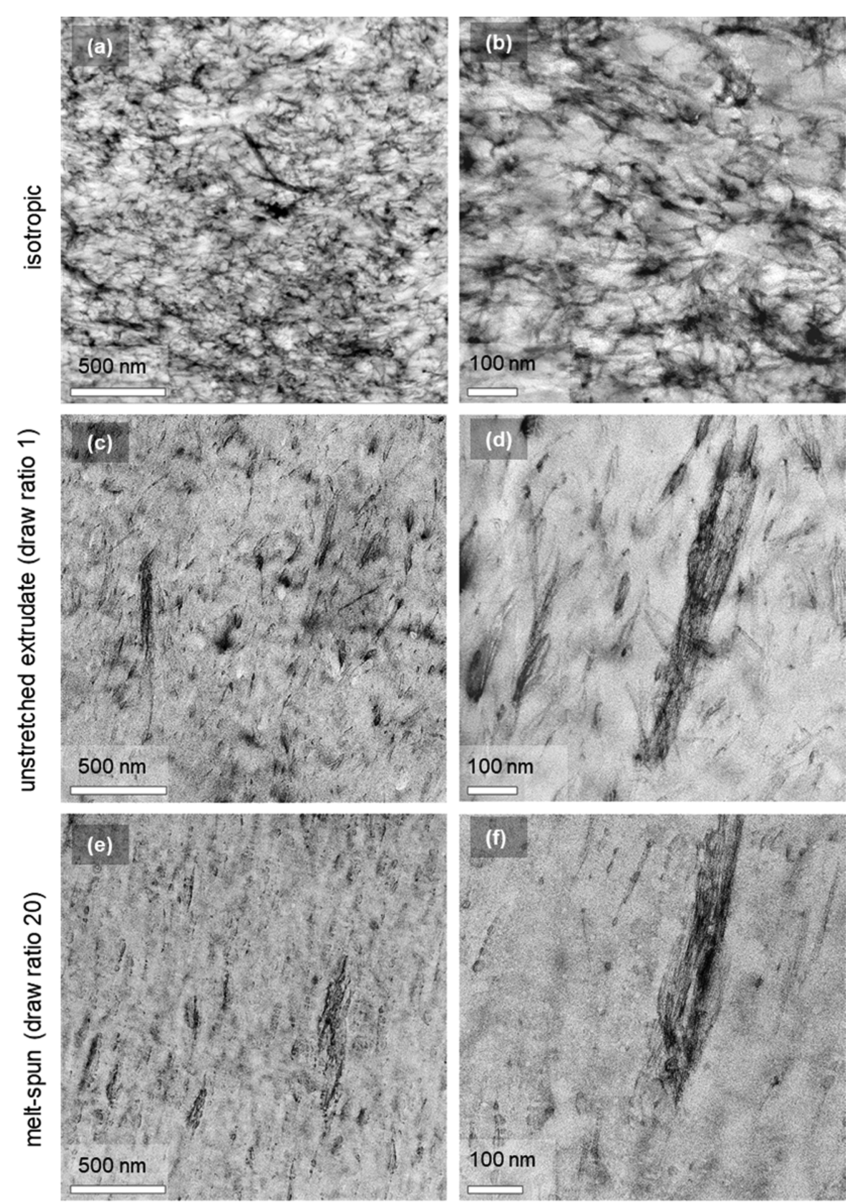

Figure 3. TEM micrographs of composites containing $10.5 \mathrm{vol} \%$ $\mathrm{CNC}$ with different levels of orientation; $(\mathrm{a}, \mathrm{b})$ isotropic composite prepared by compression molding of cast samples, $(c, d)$ unstretched extrudate, and (e, f) fibers melt-spun at a draw ratio of 20. Images were postprocessed in Adobe Photoshop for contrast enhancement (see Figure S10 for original images).

mechanical shearing during melt compounding exposes the CNC to the hydrophobic bulk of the melt, composed of the ethylene segments of $\mathrm{EAA}_{15}$, resulting in $\mathrm{CNC}$ aggregation. The CNC content, however, is not affected by melt processing as indicated by TGA thermograms of isotropic samples and melt-spun fibers with 10.5 vol \% CNC, which indicate a comparable mass loss (Figure S11).

Tensile Deformation of Anisotropic Samples. Tensile deformation experiments reveal that the elastic modulus of anisotropic samples increases monotonically with both draw ratio and $\mathrm{CNC}$ content (Figure 4). Isotropic samples show a modest increase in modulus by a factor of up to 2 (Figure $2 b$ ). Instead, fibers of $\mathrm{CNC} / \mathrm{EAA}_{15}$ with $10.5 \mathrm{vol} \% \mathrm{CNC}$, prepared with a draw ratio of 20, display a substantial increase in $E$ by almost 5 times (Figure S12), compared to drawn fibers of neat $\mathrm{EAA}_{15}$. Evidently, despite the high degree of alignment, which reduces the probability of cellulose/cellulose interactions and hence prevents the formation of a percolating network, we continue to observe a reinforcing effect.

We again compared the increase in $E$ as a function of $\mathrm{CNC}$ content with values predicted by the Halpin-Tsai model. We used a value of $E_{\mathrm{m}}=310 \mathrm{MPa}$ measured for a neat $\mathrm{EAA}_{15}$ fiber with a draw ratio of 20 and treated the anisotropic samples as symmetric around 2 axes (eq 2). The Halpin-Tsai model for 


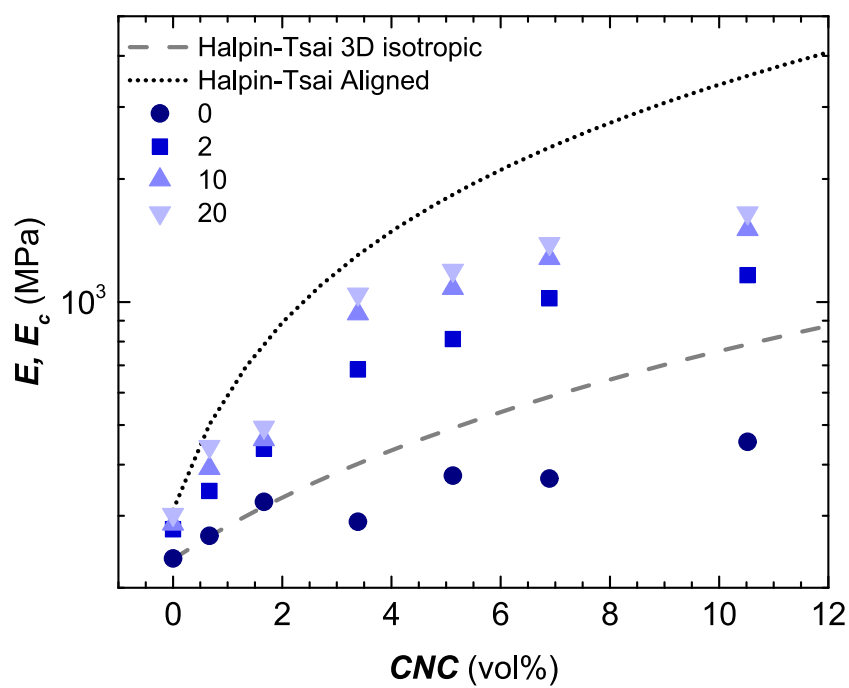

Figure 4. Young's modulus $E$ of samples melt-spun at draw ratios of 2, 10 , and 20, along with isotropic samples, as a function of CNC volume content. Predicted moduli $E_{\mathrm{c}}$ by the Halpin-Tsai model for 3D isotropic composites (gray dashed line) and aligned composites, symmetric around 2 axes (black dotted line). $E_{\mathrm{m}}=235 \mathrm{MPa}$ for the $3 \mathrm{D}$ isotropic model while $E_{\mathrm{m}}=310 \mathrm{MPa}$ for the aligned model, which are the moduli of compression molded and stretched (draw ratio of 20) $\mathrm{EAA}_{15}$, respectively.

aligned composites slightly overestimates the moduli measured for fibers prepared with a draw ratio of 20 (Figure 4), which we again explain with aggregation of CNC (Figure 3 ). The Halpin-Tsai model for aligned composites slightly overestimates the moduli measured for fibers prepared with a draw ratio of 20 (Figure 4). This is not surprising, keeping in mind that the aligned Halpin-Tsai model marks the upper bound for possible reinforcement, assuming perfect fiber alignment as well as perfect dispersion of reinforcement, both of which assumptions are not met in our composites (Figure 3). The Ouali model, in contrast, is not suitable to describe the oriented composite samples as percolation of CNC is thought to occur above the here-studied range of compositions. Provided that the $\mathrm{CNC}$ reinforcing agent does not form a percolating network, the reinforcing effect must instead occur due to stress transfer via cellulose/polymer interactions.

Cellulose/Polymer Interface Region. We used dynamic mechanical analysis to gain insight into the heterogeneity of the polymer matrix that may result from cellulose/polymer interactions. In particular, we extracted information about the glass transition temperature (s) $T_{\mathrm{g}}$ of neat $\mathrm{EAA}_{15}$ and $\mathrm{CNC} /$ $\mathrm{EAA}_{15}$ containing 10.5 vol \% CNC (Figure 5). Neat $\mathrm{EAA}_{15}$ shows a single peak in $\tan \delta$ at $-42{ }^{\circ} \mathrm{C}$, whereas the composite feature two distinct peaks, located at -49 and $-43{ }^{\circ} \mathrm{C}$. The emergence of a second $T_{\mathrm{g}}^{\prime}$ is reproducible in samples with lower cellulose loading (Figure S13). The occurrence of two distinct glass transitions suggests that two distinct types of domains exist within the polymer matrix, which we assign to bulk material and a polymer layer close to a CNC surface, respectively. The value of $-43{ }^{\circ} \mathrm{C}$ corresponds to the $T_{\mathrm{g}}$ of neat $\mathrm{EAA}_{15}$, which indicates that part of the matrix material in the composite is comparable to neat $\mathrm{EAA}_{15}$. The second peak in $\tan \delta$ at $-49^{\circ} \mathrm{C}$ occurs at a lower temperature than the $T_{\mathrm{g}}$ of neat $\mathrm{EAA}_{15}$. We argue that favorable interactions between $\mathrm{EAA}_{15}$ and $\mathrm{CNC}$ change the nanostructure of $\mathrm{EAA}_{15}$ close to the CNC surface (note that differential scanning calorimetry
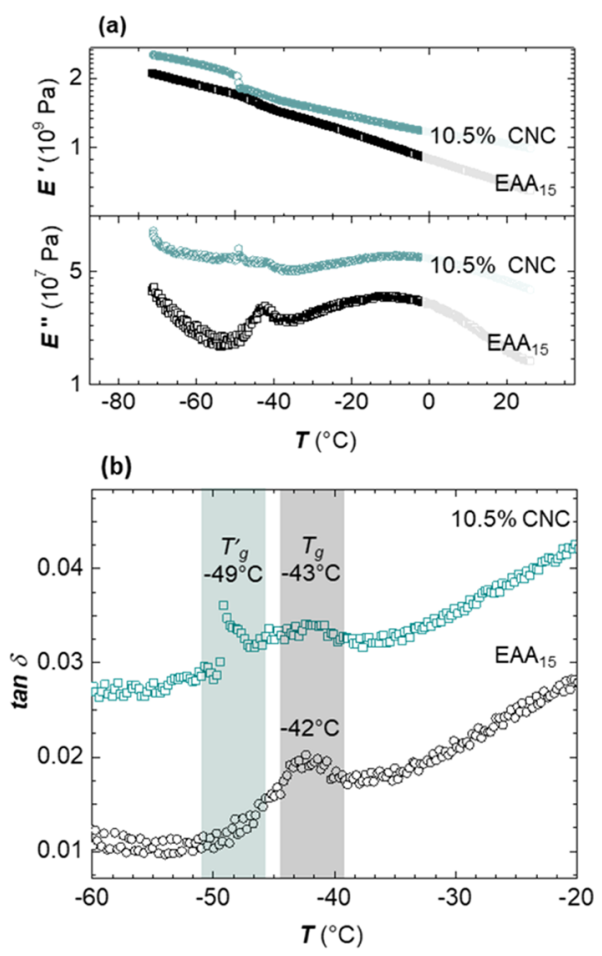

Figure 5. (a) Storage and loss moduli $E^{\prime}$ and $E^{\prime \prime}$ and (b) loss tangent $\tan \delta$ of neat $\mathrm{EAA}_{15}$ (black) and the composite with $10.5 \mathrm{vol} \% \mathrm{CNC}$ (teal) measured with DMA; $T_{\mathrm{g}}$ values were extracted from peaks of the loss tangent $\tan \delta$.

(DSC) suggests a similar crystallinity of $11 \%$; Figure S14). As a result, CNC particles are surrounded by a less dense $\mathrm{EAA}_{15}$ phase where polymer chains can relax more easily compared to those located further away from the CNC surface, resulting in a lower $T_{\mathrm{g}}^{\prime}$. This interpretation is consistent with the work by Venkatesh et al. ${ }^{29}$ who used X-ray tomography to study composites of microfibrillated cellulose and $\mathrm{EAA}_{15}$ and observed a lower density phase close to the surface of cellulose.

We used thermal gravimetric analysis (TGA) to compare the loss of volatiles such as water between 50 and $150{ }^{\circ} \mathrm{C}$ in neat $\mathrm{EAA}_{15}$ and the composite containing $10.5 \mathrm{vol} \% \mathrm{CNC}$ (Figure S15). The similar mass loss of $0.2-0.3 \%$ indicates that plasticization by water or other volatile species is unlikely to account for the presence of domains with a lower $T_{\mathrm{g}}^{\prime}$.

Molecular Dynamics Simulations. We carried out molecular dynamics (MD) simulations at a temperature of $140{ }^{\circ} \mathrm{C}$, i.e., above $T_{\mathrm{m}}=90{ }^{\circ} \mathrm{C}$ of the matrix, to study the $\mathrm{CNC} / \mathrm{EAA}_{15}$ interface in more detail. In our simulations, $\mathrm{EAA}_{15}$ was treated as a linear copolymer with sodium acrylate units statistically distributed along the chain, in line with the comonomer distribution inferred from fractional crystallization (Figure S2). We created 10 different linear chain models of $\mathrm{EAA}_{15}$, each with a degree of polymerization (DP) of 64, which differed only in the positions of the carboxylate groups along the backbone. This oligomeric model of $\mathrm{EAA}_{15}$ is here forth referred to as the ionomer. For CNC, a model of the $\mathrm{I} \beta$ allomorph of cellulose was created by arranging $5 \times 5$ cellulose chains in a cross-sectional rectangular shape, each chain having a DP of 20 (Figure S16). The primary surface hydroxyl groups of each chain were substituted with the sulfate half-ester groups, where seven out of ten groups were sulfonated, which corresponds to a typical experimental surface charge density of CNC corresponding to $200-335 \mu \mathrm{eq} \mathrm{g}^{-1} .^{48}$ The sulfonate 
(a)

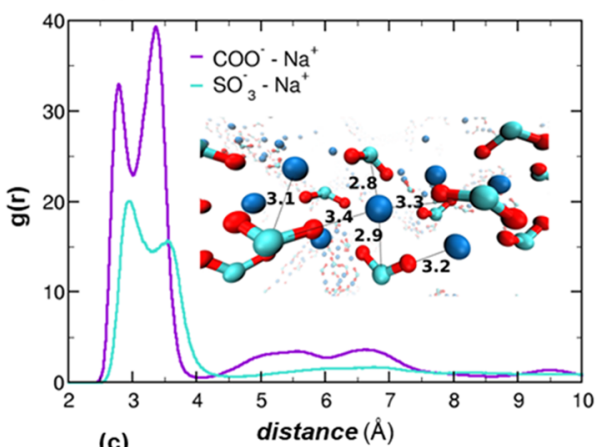

(c)

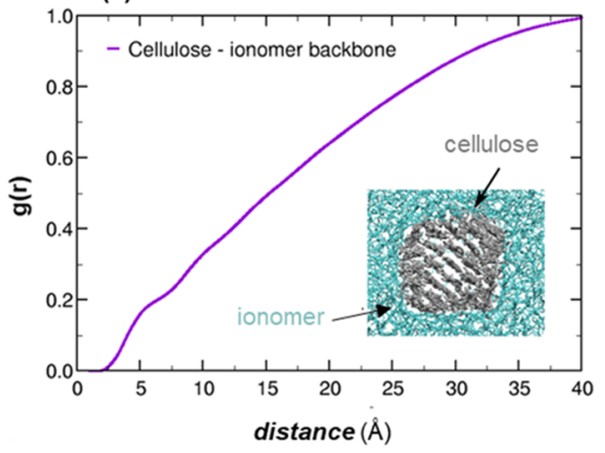

(b)

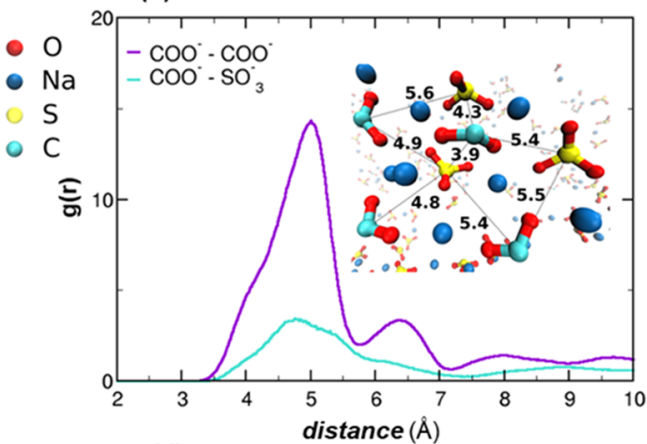

(d)

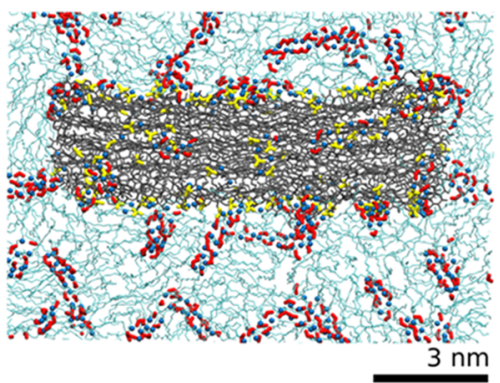

Figure 6. Radial distribution functions $g(\mathrm{r})$ between (a) anionic groups of cellulose or the simulated ionomer and $\mathrm{Na}^{+}$, (b) anionic groups of cellulose and the simulated ionomer as well as within the ionomer, and (c) cellulose and the backbone carbon atoms of the ionomer and (d) simulation snapshot of the system at the end of the simulation. The inset simulation snapshots highlight the distances, in angstrom, associated with the respective distribution functions.

(a)

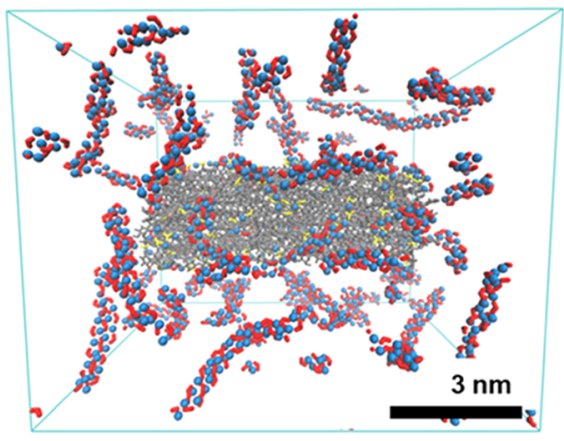

(c)

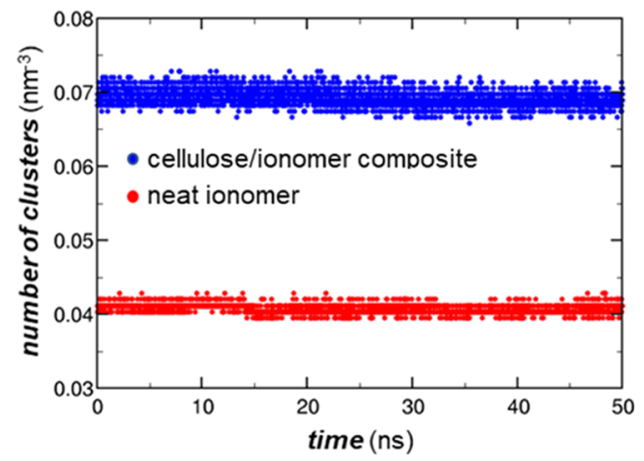

(b)

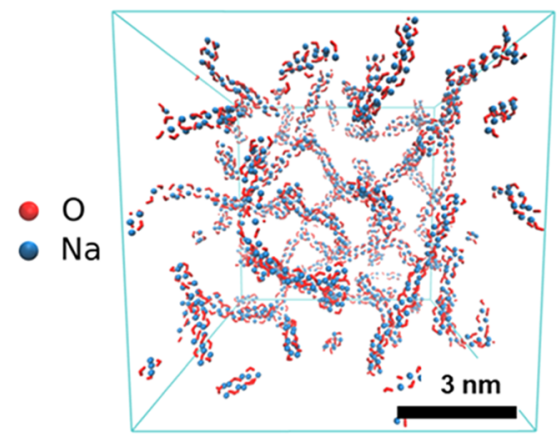

(d)

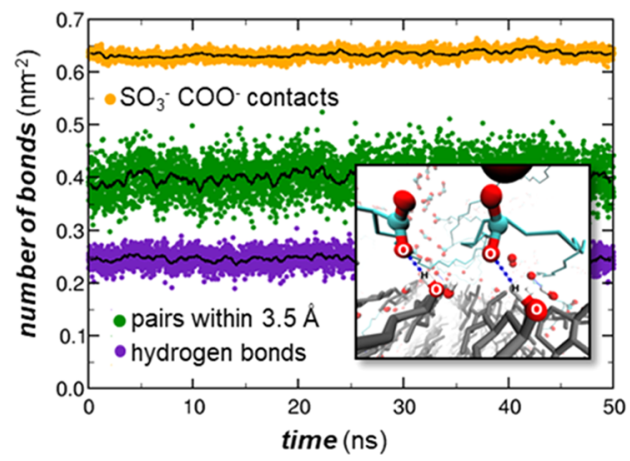

Figure 7. Snapshot of the simulated EAA ionomer (a) with cellulose and (b) without cellulose, (c) number of ionic clusters normalized to the box volume as a function of the simulation time, and (d) number of bonds between cellulose and the simulated ionomer normalized to the cellulose surface area. Hydrogen bonds and pairs of atoms available for formation of hydrogen bonds (within $3.5 \AA$ ) along with the number of contacts between $\mathrm{COO}^{-}$and $\mathrm{SO}_{3}{ }^{-}$. The black lines are running averages of the respective properties. The inset image shows hydrogen bonds (in blue) formed between the $\mathrm{COO}^{-}$groups of the polymer and the hydroxyl groups of cellulose. 
groups carry a negative charge, and therefore, we introduce an additional $126 \mathrm{Na}^{+}$ions to neutralize the system.

MD Simulations: Nature of $\mathrm{CNC} /$ lonomer Interactions. We calculated the radial distribution functions (RDFs) for various functional groups and ions to identify the main interactions between $\mathrm{CNC}$ and $\mathrm{EAA}_{15}$. More specifically, we calculated $\mathrm{RDFs}$ between carboxylate groups $\left(\mathrm{COO}^{-}\right)$and sodium ions $\left(\mathrm{Na}^{+}\right)$, between sulfonate groups $\left(\mathrm{SO}_{3}{ }^{-}\right)$and $\mathrm{Na}^{+}$ (Figure 6a), between $\mathrm{COO}^{-}$groups, between $\mathrm{COO}^{-}$and $\mathrm{SO}_{3}{ }^{-}$groups (Figure $6 \mathrm{~b}$ ), as well as between cellulose and the carbon atoms that make up the ionomer backbone (Figure 6c). It should be mentioned that for the carboxylate and sulfonate groups, we used the carbon and sulfur atoms, respectively, as reference points in the RDFs, e.g., the carboxylate-sulfonate group $\mathrm{RDF}$ is calculated between the $\mathrm{C}$ and $\mathrm{S}$ atoms. For the cellulose/polymer backbone RDF (Figure 6c), we used all atoms from cellulose as a reference.

The insets in Figure $6 a-c$ show representative simulation snapshots and configurations highlighting the distances associated with the main $\mathrm{RDF}$ peaks. The $\mathrm{Na}^{+}$cations are strongly coordinated with the $\mathrm{COO}^{-}$and $\mathrm{SO}_{3}{ }^{-}$groups, which is evident from the peaks at around $3 \AA$ (Figure 6a). The RDFs and simulation snapshot shown in Figure $6 \mathrm{~b}$ demonstrate that there is strong coordination between $\mathrm{COO}^{-}$groups, as well as between $\mathrm{COO}^{-}$and $\mathrm{SO}_{3}{ }^{-}$groups. These anionic groups would repeal each other, and their interactions therefore must be mediated through $\mathrm{Na}^{+}$ions. The cations are coordinated between two $\mathrm{COO}^{-}$or between $\mathrm{COO}^{-}$and $\mathrm{SO}_{3}{ }^{-}$groups and thus form strong complexes due to electrostatic interactions. While the $\mathrm{COO}^{-}-\mathrm{COO}^{-}$bridges give rise to ionic clusters within the $\mathrm{EAA}_{15}$, the $\mathrm{COO}^{-}-\mathrm{SO}_{3}^{-}$interactions result in adhesion of $\mathrm{EAA}_{15}$ to the cellulose surface.

Further evidence for bridging of anionic groups by $\mathrm{Na}^{+}$ions is given by the location of the RDF main peaks (Figure $6 \mathrm{~b}$ ). These peaks at about $5 \AA$ are located at larger distances compared to the peaks in Figure 6a. This means that $\mathrm{Na}^{+}$ions can be coordinated between two neighboring anionic groups, which was previously shown by density functional theory calculations. ${ }^{49}$ Lastly, the RDF between the backbone carbon atoms of the ionomer and cellulose (Figure 6c) show that there is no preferential conformation of the polymer backbone with respect to the cellulose surface, as indicated by the absence of peaks in the RDF. This confirms that the main interactions between cellulose and the ionomer are between $\mathrm{COO}^{-}$and $\mathrm{SO}_{3}{ }^{-}$groups mediated by $\mathrm{Na}^{+}$ions. The absence of interactions between cellulose and the polymer backbone also implies that the good dispersion of CNC in our composites arises because $\mathrm{EAA}_{15}$ has a relatively high amount of sodium carboxylate functional groups. $\mathrm{CNC}$ is known to aggregate in nonpolar matrices due to formation of hydrogen bonds between cellulose particles if there are no competing interactions favoring dispersion. ${ }^{35,50}$ Figure $6 \mathrm{~d}$ clearly shows that some of the $\mathrm{COO}^{-}$groups (in red) are found next to the cellulose surface and extend into the polymer matrix, which prevents CNC aggregation.

MD Simulations: CNC/lonomer Interactions Per Unit Volume. We used the results of our MD simulations to compare the nanostructure of the simulated $\mathrm{CNC} /$ ionomer composite and the neat ionomer. A comparison of simulation snapshots of these two systems (Figure 7a,b) reveals that the $\mathrm{COO}^{-}$groups together with $\mathrm{Na}^{+}$form stringlike ionic aggregates that percolate through the ionomer matrix. Similar structures have been predicted by previous MD simulations of ionomers. ${ }^{51-54}$ Figure $7 \mathrm{c}$ presents the number of clusters, normalized to the simulation box volume, of $\mathrm{COO}^{-}$groups bridged by $\mathrm{Na}^{+}$ions as a function of the simulation time. Groups are identified to belong to the same cluster if the distance between them is less than $5.7 \AA$, which corresponds to the minimum in the $\mathrm{RDF}$ of two $\mathrm{COO}^{-}$groups after the first peak in Figure $6 \mathrm{~b}$. The simulated $\mathrm{CNC} /$ ionomer composite has almost double the amount of clusters per volume $(\sim 0.07$ $\left.\mathrm{nm}^{-3}\right)$ compared with the neat ionomer $\left(\sim 0.04 \mathrm{~nm}^{-3}\right)$. Additionally, the average number of $\mathrm{COO}^{-}$groups belonging to one cluster in the $\mathrm{CNC} /$ ionomer composite is $15.3 \pm 0.2$, while clusters in the neat ionomer system comprise $24.8 \pm 0.4$ groups. These observations imply that the presence of cellulose in the polymer matrix disrupts the formation of ionic aggregates, and thus, more but smaller aggregates can be found in the $\mathrm{CNC}$ /ionomer composite. The tendency of the $\mathrm{COO}^{-}$groups to interact with the $\mathrm{SO}_{3}{ }^{-}$groups, which was shown in the RDFs (Figure 6b), also indicates a competing interaction, which likely affects cluster formation.

We investigated the number of ionic bonds, formed via interactions between $\mathrm{SO}_{3}{ }^{-}$from cellulose and $\mathrm{COO}^{-}$from the ionomer. The number of ionic contact points was normalized to the cellulose surface area. It should be noted that the cellulose surface area was calculated as the solvent-accessible surface area. Two groups are considered to be in contact if the distance between them is less than $7 \AA$, i.e., the minimum after the first peak in the $\mathrm{RDF}$ between $\mathrm{SO}_{3}{ }^{-}$and $\mathrm{COO}^{-}$(Figure $6 \mathrm{~b})$. It should be noted that $\mathrm{SO}_{3}{ }^{-}$can be in contact with more than one $\mathrm{COO}^{-}$group, which is evident from the inset snapshot in Figure $6 \mathrm{~b}$. The number of ionic contact points per surface area between $\mathrm{SO}_{3}^{-}$and $\mathrm{COO}^{-}$is found to be $\approx 0.63$ $\mathrm{nm}^{-2}$ (Figure $7 \mathrm{~d}$ ).

We also considered the possibility for formation of hydrogen bonds between $\mathrm{COO}^{-}$groups of the ionomer (hydrogen-bond acceptors) and hydroxyl groups of cellulose (hydrogen-bond donors). The total number of available pairs is specified as the number of pairs of atoms able to form hydrogen bonds between CNC and the ionomer, which are within $3.5 \AA$ distance from each other, normalized to the cellulose surface area. Of all of the available pairs able to form hydrogen bonds around $60 \%$ are bonded (Figure $7 \mathrm{~d}$ ). The total number of hydrogen bonds per unit cellulose area is $\approx 0.25 \mathrm{~nm}^{-2}$. The presence of numerous hydrogen bonds, besides ionic bonds, further enhances the interaction between $\mathrm{CNC}$ and $\mathrm{EAA}_{15}$.

Based on these findings we propose that the stress transfer in the here studied composite is mediated through a polymer layer, surrounding the $\mathrm{CNC}$ particles, which has a distinctly different nanostructure compared to the matrix further away from the CNC surface. MD simulations indicate that both the size and the number of ionic clusters are critically affected by the presence of $\mathrm{CNC}$ and that numerous ionic interactions as well as hydrogen bonding between CNC and the polymer take place. The emergence of a less dense polymer layer that surrounds CNC particles, consistent with the appearance of a second $T_{\mathrm{g}}^{\prime}$ (cf. Figure 5), arises due to favorable interactions between $\mathrm{EAA}_{15}$ and $\mathrm{CNC}$.

\section{CONCLUSIONS}

We have studied a cellulose nanocomposite composed of CNC and an ethylene-acrylate ionomer matrix. Using a combined experimental and modeling approach, we have inferred the existence of a polymer layer close to the CNC surface where ionic clusters as well as hydrogen bonding govern the 
interactions between $\mathrm{CNC}$ and $\mathrm{EAA}_{15}$. We conclude that stress transfer in the composite is mediated via these strong cellulose/polymer interactions, and that cellulose/cellulose interactions play a minor role. The importance of cellulose/ polymer interactions is confirmed by the five times higher storage modulus of melt-spun composite fibers, as compared to fibers of the neat matrix polymer since the orientation of CNC reduces the probability of cellulose/cellulose interactions.

\section{ASSOCIATED CONTENT}

\section{s) Supporting Information}

The Supporting Information is available free of charge at https://pubs.acs.org/doi/10.1021/acs.macromol.0c02305.

Transient extensional viscosity of $\mathrm{EAA}_{15}$, fractional crystallization of $\mathrm{EAA}_{15}, \mathrm{CNC}$ aspect ratio measurements, original TEM images, conversion of wt \% to vol $\%$, preparation of anisotropic fibers and calculation of melt-draw ratios, WAXS of isotropic and anisotropic composites, description and results of ROSMAS NMR spectroscopy, mass loss from TGA, normalized tensile moduli of anisotropic composites, dynamic mechanical thermal analysis (DMTA) of isotropic composites, calculation of crystallinity of $\mathrm{EAA}_{15}$, and detailed description of $\mathrm{MD}$ simulations (PDF)

\section{AUTHOR INFORMATION}

\section{Corresponding Authors}

Giada Lo Re - Department of Industrial and Materials

Science, Chalmers University of Technology, 41296

Göteborg, Sweden; ㅈorcid.org/0000-0001-8840-1172;

Email: giadal@chalmers.se

Igor Zozoulenko - Laboratory of Organic Electronics, ITN, Linköping University, 60174 Norrköping, Sweden; Wallenberg Wood Science Center, Linköping University, 581 83 Linköping, Sweden; 이이.org/0000-0002-6078-3006; Email: igor.zozoulenko@liu.se

Christian Müller - Department of Chemistry and Chemical Engineering and Wallenberg Wood Science Center, Chalmers University of Technology, 41296 Göteborg, Sweden; (1) orcid.org/0000-0001-7859-7909;

Email: christian.müller@chalmers.se

\section{Authors}

Anna Peterson - Department of Chemistry and Chemical Engineering, Chalmers University of Technology, 41296 Göteborg, Sweden

Aleksandar Y. Mehandzhiyski - Laboratory of Organic Electronics, ITN, Linköping University, 60174 Norrköping, Sweden; (1) orcid.org/0000-0001-5671-4545

Leo Svenningsson - Department of Chemistry and Chemical Engineering, Chalmers University of Technology, 41296 Göteborg, Sweden; 다이.org/0000-0002-3813-347X

Agnieszka Ziolkowska - Umeå Center for Electron Microscopy (UCEM), Department of Chemistry, Umeå University, 90187 Umeå, Sweden

Roland Kádár - Department of Industrial and Materials Science and Wallenberg Wood Science Center, Chalmers University of Technology, 41296 Göteborg, Sweden

Anja Lund - Department of Chemistry and Chemical Engineering, Chalmers University of Technology, 41296 Göteborg, Sweden
Linda Sandblad - Umeå Center for Electron Microscopy (UCEM), Department of Chemistry, Umeå University, 901 87 Umeå, Sweden

Lars Evenäs - Department of Chemistry and Chemical Engineering and Wallenberg Wood Science Center, Chalmers University of Technology, 41296 Göteborg, Sweden; (1) orcid.org/0000-0002-6580-0610

Complete contact information is available at: https://pubs.acs.org/10.1021/acs.macromol.0c02305

\section{Notes}

The authors declare no competing financial interest.

\section{ACKNOWLEDGMENTS}

We acknowledge financial support from the Swedish Foundation for Strategic Research (grant agreement no. RMA15-0052), the Swedish Research Council Formas (grant agreement no. FR-2018/0010), and Treesearch. G.L.R. acknowledges the Genie initiative funded by the Chalmers University of Technology Foundation for financial support. We acknowledge the facilities and technical assistance of the Umeå Center for Electron Microscopy (UCEM), supported by Swedish Research Council, project id 2018-06487 RFI NanoSPAM. We acknowledge the facilities and technical assistance of the Chalmers Materials Analysis Laboratory (CMAL). The solid-state NMR measurements were carried out at the NMR Core Facility at Umeå University, Sweden, with the assistance of Tobias Sparrman. The computations were performed on resources provided by the Swedish National Infrastructure for Computing (SNIC) at NSC and HPC2N. We thank Amir Masoud Pourrahimi for help with image editing and Anna I. Hofmann for help with photography.

\section{REFERENCES}

(1) Siqueira, G.; Bras, J.; Dufresne, A. Cellulosic Bionanocomposites: A review of preparation, properties and applications. Polymers 2010, 2, 728-765.

(2) Berglund, L. A.; Peijs, T. Cellulose biocomposites-from bulk moldings to nanostructured systems. MRS Bull. 2010, 35, 201-207.

(3) Oksman, K.; Aitomäki, Y.; Mathew, A. P.; Siqueira, G.; Zhou, Q.; Butylina, S.; Tanpichai, S.; Zhou, X. J.; Hooshmand, S. Review of the recent developments in cellulose nanocomposite processing. Composites, Part A 2016, 83, 2-18.

(4) Hao, W.; Wang, M.; Zhou, F.; Luo, H.; Xie, X.; Luo, F.; Cha, R. A review on nanocellulose as a lightweight filler of polyolefin composites. Carbohydr. Polym. 2020, 243, No. 116466.

(5) Wohlhauser, S.; Delepierre, G.; Labet, M.; Morandi, G.; Thielemans, W.; Weder, C.; Zoppe, J. O. Grafting polymers from cellulose nanocrystals: Synthesis, properties, and applications. Macromolecules 2018, 51, 6157-6189.

(6) Habibi, Y. Key advances in the chemical modification of nanocelluloses. Chem. Soc. Rev. 2014, 43, 1519-1542.

(7) Chen, J.; Lin, N.; Huang, J.; Dufresne, A. Highly alkynylfunctionalization of cellulose nanocrystals and advanced nanocomposites thereof via click chemistry. Polym. Chem. 2015, 6, 4385-4395.

(8) Chen, B.; Evans, J. R. G. Elastic moduli of clay platelets. Scr. Mater 2006, 54, 1581-1585.

(9) Dufresne, A. Cellulose nanomaterial reinforced polymer nanocomposites. Curr. Opin. Colloid Interface Sci. 2017, 29, 1-8.

(10) Favier, V.; Canova, G. R.; Shrivastava, S. C.; Cavaille, J. Y. Mechanical percolation in cellulose whisker nanocomposites. Polym. Eng. Sci. 1997, 37, 1732-1739. 
(11) Favier, V.; Canova, G. R.; Cavaille, J. Y.; Chanzy, H.; Dufresne, A.; Gauthier, C. Nanocomposite materials from latex and cellulose whiskers. Polym. Adv. Technol. 1995, 6, 351-355.

(12) Annamalai, P. K.; Dagnon, K. L.; Monemian, S.; Foster, E. J.; Rowan, S. J.; Weder, C. Water-responsive mechanically adaptive nanocomposites based on styrene-butadiene rubber and cellulose nanocrystals: Processing matters. ACS Appl. Mater. Interfaces 2014, 6, 967-976.

(13) Capadona, J. R.; Shanmuganathan, K.; Tyler, D. J.; Rowan, S. J.; Weder, C. Stimuli-responsive polymer nanocomposites inspired by the sea cucumber dermis. Science 2008, 319, 1370-1374.

(14) Shanmuganathan, K.; Capadona, J. R.; Rowan, S. J.; Weder, C. Biomimetic mechanically adaptive nanocomposites. Prog. Polym. Sci. 2010, 35, 212-222.

(15) Svagan, A. J.; Samir, M. A. S. A.; Berglund, L. A. Biomimetic polysaccharide nanocomposites of high cellulose content and high toughness. Biomacromolecules 2007, 8, 2556-2563.

(16) Juntaro, J.; Ummartyotin, S.; Sain, M.; Manuspiya, H. Bacterial cellulose reinforced polyurethane-based resin nanocomposite: A study of how ethanol and processing pressure affect physical, mechanical and dielectric properties. Carbohydr. Polym. 2012, 87, 2464-2469.

(17) Littunen, K.; Hippi, U.; Saarinen, T.; Seppälä, J. Network formation of nanofibrillated cellulose in solution blended poly(methyl methacrylate) composites. Carbohydr. Polym. 2013, 91, 183-190.

(18) Petersson, L.; Kvien, I.; Oksman, K. Structure and thermal properties of poly(lactic acid)/cellulose whiskers nanocomposite materials. Compos. Sci. Technol. 2007, 67, 2535-2544.

(19) Pereda, M.; El Kissi, N.; Dufresne, A. Extrusion of polysaccharide nanocrystal reinforced polymer nanocomposites through compatibilization with poly(ethylene oxide). ACS Appl. Mater. Interfaces 2014, 6, 9365-9375.

(20) Gopalan Nair, K.; Dufresne, A.; Gandini, A.; Belgacem, M. N. Crab shell chitin whiskers reinforced natural rubber nanocomposites. 3. Effect of chemical modification of chitin whiskers. Biomacromolecules 2003, 4, 1835-1842.

(21) Aitomäki, Y.; Oksman, K. Reinforcing efficiency of nanocellulose in polymers. React. Funct. Polym. 2014, 85, 151-156.

(22) Bondeson, D.; Oksman, K. Dispersion and characteristics of surfactant modified cellulose whiskers nanocomposites. Compos. Interfaces 2007, 14, 617-630.

(23) Lo Re, G.; Engström, J.; Wu, Q.; Malmström, E.; Gedde, U. W.; Olsson, R. T.; Berglund, L. Improved cellulose nanofibril dispersion in melt-processed polycaprolactone nanocomposites by a latex-mediated interphase and wet feeding as LDPE alternative. ACS Appl. Nano Mater. 2018, 1, 2669-2677.

(24) Grunert, M.; Winter, W. T. Nanocomposites of cellulose acetate butyrate reinforced with cellulose nanocrystals. J. Polym. Environ. 2002, 10, 27-30.

(25) Lönnberg, H.; Fogelström, L.; Samir, M. A. S. A..; Berglund, L.; Malmström, E.; Hult, A. Surface grafting of microfibrillated cellulose with $\operatorname{poly}(\varepsilon$-caprolactone $)$ - Synthesis and characterization. Eur. Polym. J. 2008, 44, 2991-2997.

(26) Garg, M.; Linares, M.; Zozoulenko, I. Theoretical rationalization of self-assembly of cellulose nanocrystals: effect of surface modifications and counterions. Biomacromolecules 2020, 21, 30693080.

(27) Meng, X.; Bocharova, V.; Tekinalp, H.; Cheng, S.; Kisliuk, A.; Sokolov, A. P.; Kunc, V.; Peter, W. H.; Ozcan, S. Toughening of nanocelluose/PLA composites via bio-epoxy interaction: Mechanistic study. Mater. Des. 2018, 139, 188-197.

(28) Peterson, A.; Östergren, I.; Lotsari, A.; Venkatesh, A.; Thunberg, J.; Ström, A.; Rojas, R.; Andersson, M.; Berglund, L. A.; Boldizar, A.; Müller, C. Dynamic nanocellulose networks for thermoset-like yet recyclable plastics with a high melt stiffness and creep resistance. Biomacromolecules 2019, 20, 3924-3932.

(29) Venkatesh, A.; Thunberg, J.; Moberg, T.; Klingberg, M.; Hammar, L.; Peterson, A.; Müller, C.; Boldizar, A. Cellulose nanofibril-reinforced composites using aqueous dispersed ethyleneacrylic acid copolymer. Cellulose 2018, 25, 4577-4589.
(30) Keating, M. Y.; McCord, E. F. Evaluation of the comonomer distribution in ethylene copolymers using DSC fractionation. Thermochim. Acta 1994, 243, 129-145.

(31) Damm, W.; Frontera, A.; Tirado-Rives, J.; Jorgensen, W. L. OPLS all-atom force field for carbohydrates. J. Comput. Chem. 1997, $18,1955-1970$.

(32) Jorgensen, W. L.; Maxwell, D. S.; Tirado-Rives, J. Development and testing of the OPLS all-atom force field on conformational energetics and properties of organic liquids. J. Am. Chem. Soc. 1996, $118,11225-11236$.

(33) Abraham, M. J.; Murtola, T.; Schulz, R.; Páll, S.; Smith, J. C.; Hess, B.; Lindahl, E. GROMACS: High performance molecular simulations through multi-level parallelism from laptops to supercomputers. SoftwareX 2015, 1-2, 19-25.

(34) Affdl, J. C. H.; Kardos, J. L. The Halpin-Tsai equations - A Review. Polym. Eng. Sci. 1976, 16, 344-352.

(35) Dufresne, A. Cellulose nanomaterials as green nanoreinforcements for polymer nanocomposites. Philos. Trans. R. Soc., A 2018, 376, No. 20170040.

(36) van Es, M.; Feng, X. Q.; van Turnhout, J.; van der Giessen, E. Specialty Polymer Additives: Principles and Applications; John Wiley \& Son, Hoboken, NJ, 2001.

(37) Ouali, N.; Cavaillé, J. Y.; Perez, J. Elastic, viscoelastic and plastic behavior of multiphase polymer blends. Plast., Rubber Compos. Process. Appl. 1991, 16, 55-60.

(38) Takayanagi, M.; Uemura, S.; Minami, S. Application of equivalent model method to dynamic rheo-optical properties of crystalline polymer. J. Polym. Sci., Part C: Polym. Symp. 2007, 5, 113122.

(39) Pötschke, P.; Brünig, H.; Janke, A.; Fischer, D.; Jehnichen, D. Orientation of multiwalled carbon nanotubes in composites with polycarbonate by melt spinning. Polymer 2005, 46, 10355-10363.

(40) Du, F. M.; Fischer, J. E.; Winey, K. I. Effect of nanotube alignment on percolation conductivity in carbon nanotube/polymer composites. Phys. Rev. B 2005, 72, No. 121404.

(41) Qu, M.; Milsson, F.; Schubert, D. W. Effect of filler orientation on the electrical conductivity of carbon fiber/PMMA composites. Fibers 2018, 6, 3.

(42) Svenningsson, L.; Sparrman, T.; Bialik, E.; Bernin, D.; Nordstierna, L. Molecular orientation distribution of regenerated cellulose fibers investigated with rotor synchronized solid state NMR spectroscopy. Cellulose 2019, 26, 4681-4692.

(43) Harbison, G. S.; Vogt, V.-D.; Spiess, H. W. Structure and order in partially oriented solids - characterization by $2 \mathrm{D}$-magic-anglespinning NMR. J. Chem. Phys. 1987, 86, 1206-1218.

(44) Dufresne, A. Processing of Nanocellulose-Based Materials. In Nanocellulose; De Gruyter, Berlin/Boston, 2017; pp 351-418.

(45) Alloin, F.; D’Aprea, A.; Dufresne, A.; El Kissi, N.; Bossard, F. Poly(oxyethylene) and ramie whiskers based nanocomposites: influence of processing: extrusion and casting/evaporation. Cellulose 2011, 18, 957-973.

(46) Sapkota, J.; Kumar, S.; Weder, C.; Foster, J. E. Influence of processing conditions on properties of poly(vinyl acetate)/cellulose nanocrystal nanocomposites. Macromol. Mater. Eng. 2015, 300, 562571.

(47) Hietala, M.; Mathew, A. P.; Oksman, K. Bionanocomposites of thermoplastic starch and cellulose nanofibers manufactured using twin-screw extrusion. Eur. Polym. J. 2013, 49, 950-956.

(48) Reid, M. S.; Villalobos, M.; Cranston, E. D. The role of hydrogen bonding in non-ionic polymer adsorption to cellulose nanocrystals and silica colloids. Curr. Opin. Colloid Interface Sci. 2017, 29, 76-82.

(49) Mehandzhiyski, A. Y.; Riccardi, E.; van Erp, T. S.; Koch, H.; Åstrand, P. O.; Trinh, T. T.; Grimes, B. A. Density functional theory study on the interactions of metal ions with long chain deprotonated carboxylic acids. J. Phys. Chem. A 2015, 119, 10195-10203.

(50) Eichhorn, S. J.; Dufresne, A.; Aranguren, M.; Marcovich, N. E.; Capadona, J. R.; Rowan, S. J.; Weder, C.; Thielemans, W.; Roman, M.; Renneckar, S.; Gindl, W.; Veigel, S.; Keckes, J.; Yano, H.; Abe, K.; 
Nogi, M.; Nakagaito, A. N.; Mangalam, A.; Simonsen, J.; Benight, A. S.; Bismarck, A.; Berglund, L. A.; Peijs, T. Review: Current international research into cellulose nanofibres and nanocomposites. J. Mater. Sci. 2010, 45, 1-33.

(51) Bolintineanu, D. S.; Stevens, M. J.; Frischknecht, A. L. Atomistic simulations predict a surprising variety of morphologies in precise ionomers. ACS Macro Lett. 2013, 2, 206-210.

(52) Bolintineanu, D. S.; Stevens, M. J.; Frischknecht, A. L. Influence of cation type on ionic aggregates in precise ionomers. Macromolecules 2013, 46, 5381-5392.

(53) Buitrago, C. F.; Bolintineanu, D. S.; Seitz, M. E.; Opper, K. L.; Wagener, K. B.; Stevens, M. J.; Frischknecht, A. L.; Winey, K. I. Direct comparisons of X-ray scattering and atomistic molecular dynamics simulations for precise acid copolymers and ionomers. Macromolecules 2015, 48, 1210-1220.

(54) Frischknecht, A. L.; Winey, K. I. The evolution of acidic and ionic aggregates in ionomers during microsecond simulations. J. Chem. Phys. 2019, 150, No. 064901. 